FY19-FY20 Hospital Acquired Pressure Injury (HAPI) Rate Patient Demographics (Pediatric only)

Median Household Income among Patients with California Zip Codes (95,883 Patient Days 57\% of total)

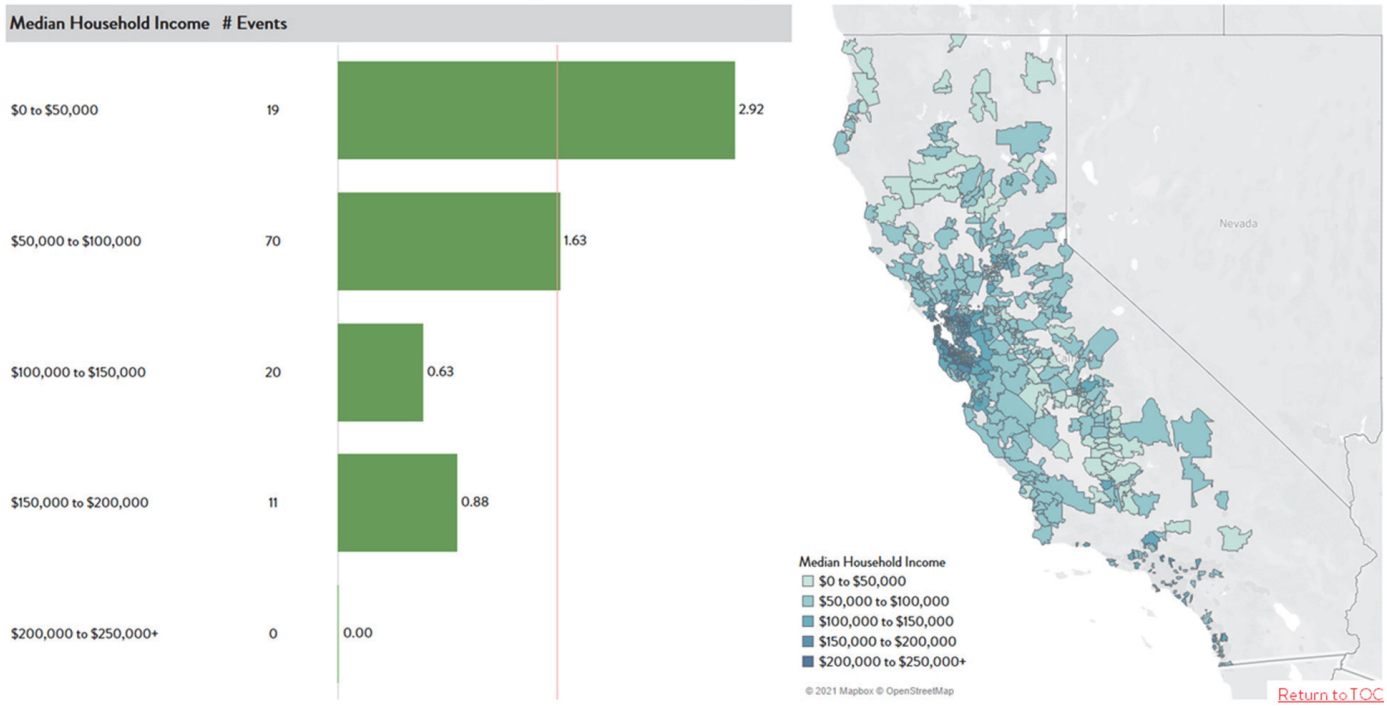

Abstract 20 Figure 1 Left shows the rate of hospital acquired pressure injuries separated by median household income. Right shows median household income by county for counties from which a patient visited our health system

improvement initiatives that seek to better understand and decrease the influence of these factors.

\section{IMPLEMENTING VIRTUAL CARE PLANNING WITH RESIDENT/FAMILY: A CANADIAN COVID LONG-TERM CARE EXPERIENCE}

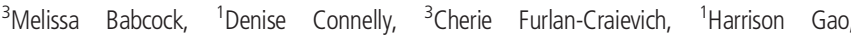
${ }^{1}$ Anna Garnett, ${ }^{7}$ Pam Hamilton, ${ }^{1}$ Melissa Hay, ${ }^{2}$ Lillian Hung, ${ }^{4}$ Jacqueline Ripley, ${ }^{6}$ Samantha Salatino, ${ }^{5}$ Lori Schindel Martin, ${ }^{4}$ Shannon Snelgrove, ${ }^{6}$ Nancy Snobelen. ${ }^{1}$ Western University, London, Canada; ${ }^{2}$ University of British Columbia, Vancouver, Canada; ${ }^{3}$ Vision 74, Sarnia, Canada; ${ }^{4}$ Copper Terrace, Chatham-Kent, Canada; ${ }^{5}$ Ryerson University, Toronto, Canada; ${ }^{6}$ WeRPN, Toronto, Canada; ${ }^{7}$ PIECES Canada, Toronto, Canada

\subsection{6/bmjoq-2021-|HI.21}

Background COVID-19 imposed extreme constraints on Canadian long-term care (LTC) homes, leading to intense isolation for residents, restricted family visits, and staff shortages. Consequently, these challenges negatively impacted the mental and physical health of residents, family, and interdisciplinary workforce in LTC homes.

Objectives

1. To describe how two LTC homes addressed promising practices - Presence of family, People in the workforce, and Future COVID-19 and non-COVID-19 care with implementation science.

2. To understand the enablers, barriers and outcomes to the implementation of an innovative re-engineered intervention P.I.E.C.E.S. ${ }^{\mathrm{TM}}$ (PIECES), designed to include families virtually for team-based resident care planning, and empower Registered Practical Nurses (RPNs) to build resilience and wellbeing.

Methods An interdisciplinary team (residents and family, nurses, and academic researchers), guided by the Consolidated Framework for Implementation Research, employed a mixedmethod design to investigate implementation processes, determinants, and outcomes. Interviews with residents/families and staff focus groups provided insight into enablers and challenges. Pre- and post-intervention results of the Connor Davidson Resilience Scale, Resilience at Work, and Assessment of Interprofessional Team Collaboration Scale informed understandings of personal, professional and organizational resilience outcomes.

Results Findings highlighted how implementation of virtual PIECES helped sustain enhanced person-centered care through more comprehensive, collaborative, efficient and effective teams, leading to better outcomes for residents. Moreover, results suggested improved resilience, wellbeing, and communication between LTC home staff and family. New learnings improved preparedness for future outbreaks. Providing a plan for adapting, embedding, and sustaining the intervention based on implementation science will accelerate the spread of highquality actionable research evidence.

Conclusions This is the first study to explore implementation processes of a virtually delivered PIECES intervention with meaningful engagement of multiple stakeholders (residents, family, RPNs). Findings provide evidence supporting important healthcare improvements, future spread of virtual interventions, and practice and policy changes for the LTC home sector.

\section{WELL BEGUN IS HALF DONE}

${ }^{1}$ Jason Boulanger, ${ }^{2}$ Evan Benjamin, ${ }^{2}$ Rachel Moyal-Smith, ${ }^{2}$ Patricia Folcarelli. ${ }^{1}$ CRICO; ${ }^{2}$ Ariadne Labs

\subsection{6/bmjoq-2021-IHI.22}

Background While many patient safety initiatives have successfully reduced harm in individual facilities and organizations, implementation, sustainment, and spread of these initiatives lags across the healthcare system.

Objectives To address this implementation gap, we created the Patient Safety Adoption framework, identifying and providing guidance for achieving the key elements of implementation (figure 1).

Methods The Framework for Implementation describes the required strategy and structure to achieve effective 